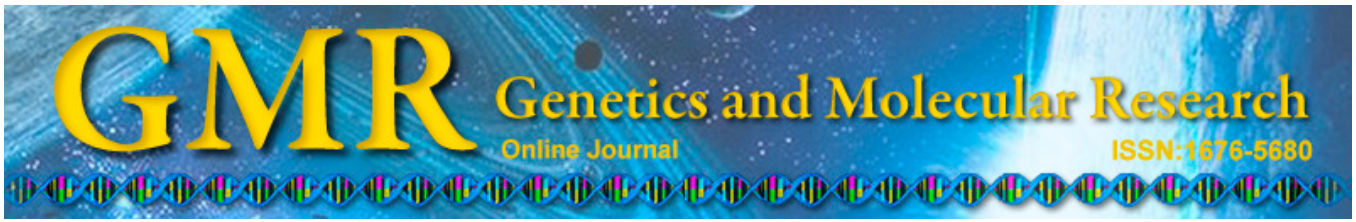

\title{
High-resolution color doppler ultrasound examination and related risk factor analysis of lower extremity vasculopathy in type 2 diabetes patients
}

\author{
J. Xu, G. Wang, D. Fu, N. Su, L. Wang, F. Gao and N. Guo \\ Second Division, Department of Endocrinology, \\ Hebei Cangzhou Central Hospital, Hebei, China \\ Corresponding author: J. Xu \\ E-mail: jxgycn@163.com
}

Genet. Mol. Res. 14 (2): 3939-3947 (2015)

Received May 22, 2014

Accepted October 8, 2014

Published April 27, 2015

DOI http://dx.doi.org/10.4238/2015.April.27.8

\begin{abstract}
This study aims to investigate the value of highresolution color Doppler ultrasonography (HR-CDU) in the evaluation of lower-extremity vasculopathy (LEV) and its related risk factors in type 2 diabetes mellitus (T2DM) patients. Two hundred forty T2DM patients were selected, who underwent lower-extremity arterial HRCDU. The patients were divided into the LEV group (V group) and the nonvasculopathy group (A group). The $\mathrm{V}$ group was then divided into the mild (B group), moderate (C group), and severe (D group) subgroups. The relevant clinical parameters were simultaneously recorded. The results showed that the lesion-positive detection rate of HR-CDU was significantly higher than that of clinical examination. The age, disease duration, smoking history, blood pressure, blood sugar, fibrinogen (FIB), C-reactive protein (CRP), uric acid (UA), and urinary albumin excretion (UAE) in the V group were higher than in the $\mathrm{N}$ group. In the logistic regression analysis, smoking history, age,
\end{abstract}


disease duration, FIB, UA, and fasting blood glucose were independent risk factors of T2DM LEV. The incidence of LEV in T2DM patients increased significantly with increasing age, UA, FIB, CRP, UAE, disease duration, and smoking history, and the vasculopathy level became more severe. In conclusion, age, disease duration, smoking history, blood pressure, blood sugar, FIB, CRP, UA, and UAE are the related risk factors of $\mathrm{LEV}$ in $\mathrm{T} 2 \mathrm{DM}$ patients.

Key words: Type 2 diabetes; Lower extremity vasculopathy; Color Doppler ultrasound; Risk factors

\section{INTRODUCTION}

As the dietary habits and lifestyle of the population change, the incidence of diabetes mellitus (DM) shows a clear upward trend and DM has become a worldwide disease. Lowerextremity arterial vasculopathy (LEAV), also known as peripheral vasculopathy, is the main cause of lower-limb amputation in DM patients, and its incidence rate is 20 times higher in DM than in non-DM patients (Pollanen et al., 2001). LEAV often occurs long before the clinical symptoms become apparent. In the early stage, lower-extremity vasculopathy (LEV) mainly shows intermittent claudication; however, if the disease exacerbates, such as in association with infections and neural lesions, local gangrene might develop, which gradually progresses to a diabetic foot ulceration (Engela et al., 2012). The treatment is rather difficult and most treatment options are traumatic, even involving amputation, which would seriously affect the patient's working ability and life quality. Therefore, LEAV is a major cause of death and disability in DM patients. If it could be detected and treated earlier, then its mortality and the amputation rates would be significantly reduced.

Previously, the evaluation of LEAV in diabetic patients mainly relies on angiography. As angiography is an invasive examination method, most patients are reluctant to undergo this procedure, thus resulting in the development of vascular lesions, failure of timely and accurate diagnosis, delay in treatment, and ultimately gangrene formation in the lower limb. This study was conducted in patients with a clinical diagnosis of type 2 DM (T2DM). High-resolution ultrasonography of the lower-limb arteries was performed to observe lower-limb arterial lesions. To evaluate whether ultrasound can aid in the early diagnosis of lower-extremity vascular disease, diabetes and clinical data were analyzed to determine the related risk factors of lower-extremity vascular disease. We also tried to provide reference data for the prevention and treatment of vascular lesions in DM patients.

\section{MATERIAL AND METHODS}

\section{Subjects}

Two hundred forty T2DM patients (108 women and 132 men) admitted at our hospital from October 2006 to December 2007 were selected. All patients met the DM diagnostic criteria developed by the World Health Organization in 1999. 


\section{Methods and indicators}

\section{General information}

The patients' gender, age, blood pressure, smoking history, DM duration and family history, presence of LEV clinical symptoms (including pain at rest, cold sensation in the lower limb, intermittent claudication, disappearing dorsalis pedis arterial pulse, and delayed healing of foot ulcers), and other parameters were recorded. Furthermore, the height and weight were measured for the calculation of body mass index.

\section{High-resolution color Doppler ultrasonography (HR-CDU) examination}

The VIDID7 imaging color Doppler diagnostic apparatus (GE, USA) was used, with the probe frequency at $13 \mathrm{MHz}$. The operation was performed by the same specialist, with the patient was in the supine or prone position. The probe was placed in the groin, scanning from top to bottom, and a bilateral comparison was done. The examination sites were the common femoral artery, femoral artery bifurcation, popliteal artery, posterior tibial artery, and dorsalis pedis artery. The observation was focused on whether the vascular intima was thickened, hardened, and showing plaque and stenosis formation. LEV was divided into four categories according to characteristics, and scored according to severity, as follows: 1) arterial intima thickness: normal $(<1 \mathrm{~mm})$, recorded as 0 point; mildly thickened $(1-1.2 \mathrm{~mm})$, recorded as 1 point; severely thickened ( $>1.2 \mathrm{~mm}$ ), recorded as 2 points. 2) Hardening: normal, recorded as 0 point; mildly hardened (the intima was not thickened, the echo was increased, and without plaque), recorded as 1 point; moderately to severely hardened (mildly hardened, associated with plaque or stenosis), recorded as 2 points. 3) Plaque: normal (no plaque formation), recorded as 0 point; single plaque, recorded as 1 point, multiple plaques, recorded as 2 points; diffused plaques, recorded as 3 points. 4) Stenosis: normal, recorded as 0 point; mild stenosis (narrowing by 30 to $50 \%$ ), recorded as 1 point; moderate or severe stenosis (narrowing by 50 to $75 \%$ ), recorded as 2 points; occlusion (no blood flow), recorded as 3 points.

The severity was assessed with the total scores as follows: 1) 0 point, normal; 2) $<10$ points, mild; 3) 10-20 points, moderate; and 4) $>20$ points, severe.

In this experiment, A represented the nonvasculopathy group (98 cases), B represented the mild group ( 23 cases), $C$ represented the moderate group (43 cases), and D represented the severe group ( 76 cases).

\section{Laboratory examination}

Before the examination, the patients fasted for $10-12 \mathrm{~h}$, then venous blood was extracted for the determination of fasting blood glucose (FBG), cholesterol, triglyceride, highdensity lipoprotein, low-density lipoprotein, fasting insulin (FINS), blood urea nitrogen, creatinine, uric acid (UA), fibrinogen (FIB), and C-reactive protein (CRP). Two hours after the administration of $75 \mathrm{~g}$ glucose, the 2-h postprandial blood glucose (2hPBG) and 2-h postprandial insulin (PINS) were determined. The blood sample was analyzed simultaneously for glycosylated hemoglobin (HbA1c), and 24-h urine was collected for the detection of the albumin excretion (UAE) rate. The insulin resistance index (HOMA-IR) was calculated according to the following formula: HOMA-IR $=$ FBG $(\mathrm{mM}) \times$ FINS $(\mathrm{mU} / \mathrm{L}) / 22.5$. The DCA2000 + 
HbA1c meter (Germany) was used to determine the HbA1c. The CA-1500 coagulation analyzer (Japan) was used to measure the FIB. The IMMULITE chemiluminescence apparatus (USA) was used to measure the UAE, FINS, and PINS, whereas the other indicators were determined with a Hitachi 7600 automatic biochemical analyzer (Japan).

\section{Statistical methods}

The SPSS11.5 statistical software package was used for statistical analysis. Normally distributed measurement data are reported as means $\pm \mathrm{SD}$; for the intergroup comparisons, the $t$-test was used. Nonnormally distributed measurement data are reported as the median and 25 to $75 \%$ percentiles; for the intergroup comparison, a nonparametric test was used. The counting data were analyzed with the chi-square test. For the correlation of LEV with other factors, logistic regression analysis was done. A P value of $<0.05$ was considered to be statistically significant.

\section{RESULTS}

\section{Comparison of the detection rate between ultrasound and clinical symptoms of diabetic lower-extremity vascular disease}

The lesion-positive detection rate of HR-CDU (59.17\%) was higher than the clinical detection rate $(17.08 \%)(\mathrm{P}<0.01)$. Therefore, ultrasonography can detect asymptomatic lower-extremity vascular disease early.

\section{Clinical data of T2DM patients in the nonlesion (A) and lesion $(B+C+D)$ groups}

The patients' age, disease duration, smoking history, diastolic blood pressure, systolic blood pressure, FBG, 2hPBG, HbA1c, HOMA-IR, FIB, CRP, UA, and UAE in the lesion group all increased, when compared with the A group, and the difference was statistically significant $(\mathrm{P}<0.01$ or $\mathrm{P}<0.05)$ (Table 1$)$.

\section{Multivariate regression analysis of T2DM LEV}

The LEV was set as the dependent variable in the multivariate logistic regression analysis. The results showed that smoking history, FIB, UA, disease duration, age, and FBG (in the order decreasing importance) were the independent risk factors of LEV in T2DM patients (Table 2).

\section{Comparison of age, disease duration, smoking history, FIB, CRP, UAE, and UA levels with T2DM LEV levels}

According to age, the patients were divided into three subgroups: the young, middle, and aged groups. Moreover, according to disease duration, the patients were divided into three groups: group 1, $\leq 5$ years; group 2, 6-10 years; group 3,>10 years. The effects of different ages and disease durations on the levels of LEV were observed, and the results showed that with increasing age and disease duration, the incidence of LEV increased significantly, and the severity became much higher (Figure 1). 
Table 1. Comparison of general indexes and biochemical indicators (means $\pm \mathrm{SD}$ ).

\begin{tabular}{|c|c|c|c|c|}
\hline Group & $\begin{array}{l}\text { A group } \\
98 \text { cases }\end{array}$ & $\begin{array}{c}\mathrm{B}+\mathrm{C}+\mathrm{D} \text { group } \\
142 \text { cases }\end{array}$ & $\begin{array}{c}\text { Statistical amount } \\
t / \chi^{2} / z\end{array}$ & $\mathrm{P}$ \\
\hline Gender (male/female) & $56 / 42$ & $76 / 66$ & 0.307 & 0.600 \\
\hline Smoking history (yes/no) & $16 / 82$ & $77 / 65$ & 35.089 & 0.000 \\
\hline Family history (yes/no) & $35 / 63$ & $54 / 88$ & 0.133 & 0.786 \\
\hline Age (years) & $51.48 \pm 12.53$ & $59.70 \pm 11.12$ & -5.341 & 0.000 \\
\hline Disease duration (years) & $4(0.9-10.0)$ & $9(4-12)$ & -3.747 & 0.000 \\
\hline $\mathrm{SBP}(\mathrm{mmHg})$ & $130(120-140)$ & $135(120-150)$ & -3.987 & 0.000 \\
\hline $\mathrm{DBP}(\mathrm{mmHg})$ & $80(70-80)$ & $80(70-90)$ & -2.228 & 0.026 \\
\hline BMI $\left(\mathrm{kg} / \mathrm{m}^{2}\right)$ & $25.53 \pm 4.21$ & $25.61 \pm 3.34$ & -0.152 & 0.880 \\
\hline $\mathrm{HbAlC}(\%)$ & $9.29 \pm 2.00$ & $10.05 \pm 2.47$ & -2.553 & 0.011 \\
\hline $\mathrm{TG}(\mathrm{mM})$ & $1.3(0.80-2.13)$ & $1.4(0.90-2.15)$ & -0.754 & 0.451 \\
\hline $\mathrm{TC}(\mathrm{mM})$ & $5.16 \pm 1.11$ & $5.26 \pm 1.25$ & -3.863 & 0.558 \\
\hline $\mathrm{HDL}-\mathrm{C}(\mathrm{mM})$ & $0.1(1.0-1.3)$ & $1.1(0.9-1.23)$ & -1.030 & 0.303 \\
\hline LDL-C (mM) & $3.15 \pm .87$ & $3.229 \pm 0.94$ & -0.703 & 0.483 \\
\hline BUN (mM) & $4.95(3.88-6.60)$ & $5.3(4.3-6.7)$ & -1.691 & 0.091 \\
\hline $\mathrm{Cr}(\mathrm{mM})$ & $61(53-74)$ & $66.5(54-82)$ & -1.604 & 0.109 \\
\hline $\mathrm{UA}(\mu \mathrm{M})$ & $248.58 \pm 89.23$ & $289.61 \pm 74.61$ & -3.863 & 0.000 \\
\hline FIB (g/L) & $2.7(2.4-3.1)$ & $3.1(2.70-4.13)$ & -5.162 & 0.000 \\
\hline $\mathrm{UAE}(\mu \mathrm{g} / \mathrm{min})$ & $8.45(5.14-17.52)$ & $9.91(5.43-66.21)$ & -1.968 & 0.049 \\
\hline $\mathrm{CRP}(\mathrm{mg} / \mathrm{dL})$ & $0.01(0.01-0.21)$ & $0.02(0.01-1.30)$ & -3.031 & 0.002 \\
\hline$\beta_{2} \mathrm{MG}(\mathrm{ng} / \mathrm{L})$ & $84.75(41-130.75)$ & $5.5(21.25-158.50)$ & -0.134 & 0.893 \\
\hline $\mathrm{FBG}(\mathrm{mM})$ & $7.35(6.68-8.75)$ & $8(6.90-10.13)$ & -1.863 & 0.042 \\
\hline $2 \mathrm{hPBG}(\mathrm{mM})$ & $15.94 \pm 2.97$ & $16.92 \pm 3.48$ & -2.252 & 0.025 \\
\hline FINS (mU/L) & $4.05(2.28-7.48)$ & $4.7(3.28-7)$ & -1.762 & 0.078 \\
\hline PINS (mU/L) & $23(13.83-31.25)$ & $24(16.35-34)$ & -1.057 & 0.290 \\
\hline HOMA-IR & $1.17(0.86-2.72)$ & $1.78(1.15-2.7)$ & -2.407 & 0.016 \\
\hline
\end{tabular}

$\mathrm{SBP}=$ systolic blood pressure; $\mathrm{DBP}=$ diastolic blood pressure; HbA1c $=$ glycosylated hemoglobin; $\mathrm{TG}=$ triglyceride; $\mathrm{TC}=$ total cholesterol; HDL-C = high-density lipoprotein; LDL-C = low-density lipoprotein; $\mathrm{BUN}=$ blood urea nitrogen; $\mathrm{Cr}=$ creatine; $\mathrm{UA}=$ uric acid; $\mathrm{FIB}=$ fibrogen; $\mathrm{UAE}=$ albumin excretion; $\mathrm{CRP}=\mathrm{C}$-reactive protein; $\beta_{2} \mathrm{MG}=$ uric $\beta_{2}$-microglobulin; $\mathrm{FBG}=$ fasting blood glucose; $2 \mathrm{hPBG}=2$-h postprandial blood glucose; FINS = fasting insulin; PINS $=2$-h postprandial; HOMA-IR $=$ insulin resistance index.

\section{Table 2. Multiple-factor logistic regression analysis.}

\begin{tabular}{|c|c|c|c|c|}
\hline & $\begin{array}{c}\text { Regression coefficient } \\
\text { B }\end{array}$ & $\begin{array}{c}\text { Correlation coefficient } \\
\text { S.E }\end{array}$ & $\begin{array}{c}\text { Relative risk degree } \\
\text { Wald }\end{array}$ & $\mathrm{P}$ \\
\hline Constant item & -9.404 & 2.669 & 12.410 & 0.000 \\
\hline Smoking history & -2.760 & 0.470 & 35.516 & 0.000 \\
\hline Age & 0.041 & 0.020 & 4.425 & 0.035 \\
\hline Disease duration & 0.086 & 0.038 & 5.117 & 0.024 \\
\hline FIB & 0.946 & 0.275 & 18.814 & 0.001 \\
\hline UA & 0.007 & 0.002 & 8.474 & 0.004 \\
\hline FBG & 0.158 & 0.077 & 4.199 & 0.040 \\
\hline
\end{tabular}

According to the levels of FIB, CRP, UAE, and UA, the patients were divided into the normal group and the increased group for the comparison of LEV levels. The results showed that the LEV incidence significantly increased, and the severity became much higher, in the increased group $(\mathrm{P}<0.01)$ (Figure 1).

According to the history of smoking, the patients were divided into two groups for the observation of different LEV levels. The results showed that the incidence rate of LEV in the smoking group was significantly higher, and the severity was also much higher $(\mathrm{P}<0.01)$ (Figure 1). 


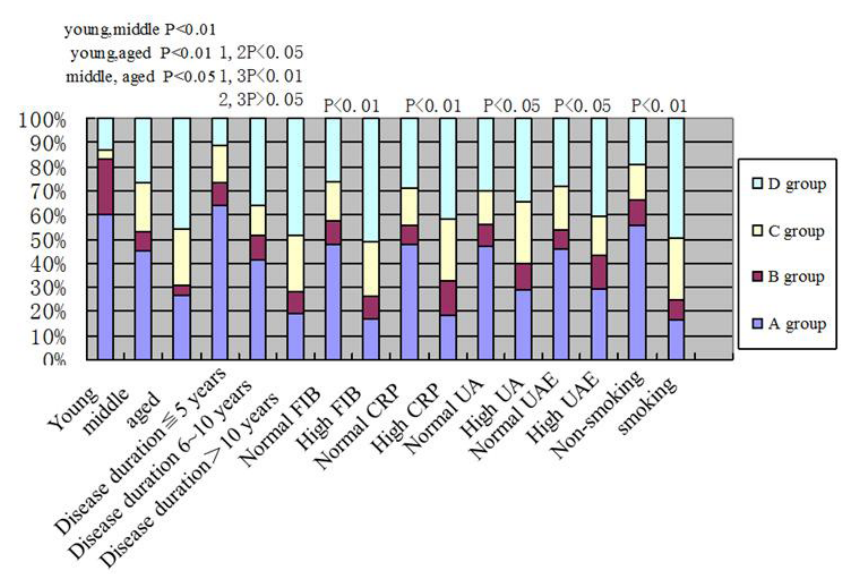

Figure 1. Comparison of age, disease duration, smoking history, FIB, CRP, UAE, and UA levels with T2DM LEV levels.

\section{DISCUSSION}

LEV in DM patients could be detected with angiography, MRI, and other methods. Although these methods are reliable, their invasive nature and high cost cause most patients to be reluctant to undergo these procedures. Thus, in many cases, LEV could not be diagnosed early and timely, leading to delay in treatment and eventually gangrene formation in severe cases and even amputation, seriously affecting the patients' work ability and life quality (Bao et al., 2011). The results of this study showed that the HR-CDU detection rate for LEV (59.17\%) was significantly higher than the clinical detection rate (17.08\%). HR-CDU is a noninvasive, simple, easy, and repeatable diagnostic tool. It could evaluate blood vessel wall thickness, hardened plaques, calcification, thrombosis, hemodynamic data, and other parameters; therefore, it could determine the location and severity of disease, and detect arterial disease in an early stage. Currently, it is considered a good noninvasive examination method, and should be routinely applied to diabetic patients for early diagnosis and treatment.

This study found that the blood pressure, age, duration of disease, and smoking history in the diabetic LEV group were significantly higher than in the nonlesion group. With increasing age and disease duration, the incidence rate of scleroderma in lower-extremity arteries also significantly increased, and the severity became much more advanced, which was consistent with a previous study (Pataky and Vischer, 2007). The multivariate regression analysis also showed that age, disease duration, and smoking history were the independent risk factors of diabetic LEV. Hypertension was also an important risk factor for LEV in T2DM patients. High blood pressure could damage the vascular endothelium, resulting in its dysfunction; thus, the permeability of blood vascular endothelium to lipoproteins would increase, increasing the generation of endothelin and leukocyte adhesion. At the same time, lipoproteins and monocyte-macrophage cells might enter the subendothelial space, promoting the formation of lipid strips. The UK Prospective Diabetes Study (UKPDS) confirmed that strict control of blood pressure could significantly reduce the incidence of peripheral vascular diseases in diabetic patients (UKPDS Group, 1998). Some studies (Makita et al., 2005) have proved that longterm smoking could cause vascular endothelial injury, increasing the levels of high-sensitivity $\mathrm{CRP}$ and thus leading to atherosclerosis. The most recent 17 prospective studies and 14 cross- 
sectional studies showed that the smoking dose was related to the incidence and development of peripheral arterial diseases, and that the prevalence of active smokers increased by 2.3 times (Willigendael et al., 2004). The older the diabetic patients, the longer the disease duration and the higher the incidence of atherosclerotic plaque. This is because high blood sugar, insulin resistance, and secondary hyperinsulinemia might directly or indirectly affect the remodeling of vascular endothelial cells and vessels, affecting vascular structures and functions. Valdivielso et al. (2007) found that smoking and disease duration were independent predictors of atherosclerotic diseases. Therefore, LEV should particularly be detected in diabetic patients with a smoking history, hypertension, long diabetes duration, and older age.

The results of this study showed that the FIB, CRP, UA, and UAE in the LEV group were significantly higher than in the A group (Table 1). Furthermore, while the incidence of LEV in the high FIB, CRP, UA and UAE subgroups significantly increased compared with the normal FIB, CRP, UA, and UAE subgroups, the severity also became much higher in the high group.

Because T2DM patients have insulin resistance, the plasminogen activator inhibitor would increase in these patients, thus inhibiting tissue plasminogen, resulting in the elevation of FIB. As an independent risk factor of diabetic LEV, FIB and its degraded products could not only directly damage the blood vessel walls, stimulating the proliferation and migration of vascular smooth muscle cells and causing endothelial dysfunction, but it can also directly activate endothelial cells and promote the expression of adhesive molecules, also leading to endothelial cell dysfunction (Seeger et al., 2002; Guo et al., 2004) and thus promoting the progress of atherosclerosis. In recent years, studies have found that the chronic low-grade inflammation state, especially with a persistent mild increase of CRP levels, was closely related to T2DM and the development of its macrovascular complications. However, the mechanism is still unclear, which might be related to insulin resistance (Zhao et al., 2007). CRP could influence endothelial cells, impairing the endothelial function and thus accelerating the progress of atherosclerosis. The prospective Physicians' Health Study suggested that a high level of CRP would increase the risk of stroke by 2-fold, the risk of myocardial infarction by 3 -fold, and the risk of peripheral vascular diseases by 1 -fold (Ridker et al., 1997). Increased serum UA levels were reported to be able to promote the development of atherosclerosis (Kanellis and Kang, 2005; Zoccali et al., 2006), and it was reported domestically that UA was an important independent risk factor for type 2 diabetes macroangiopathy. Increased UA might lead to the vascular complications, with the possible reasons as follows: high UA levels would influence traditional factors such as abnormal lipoprotein metabolism and hypertension, and promote the formation of atherosclerosis. Urate is a known inflammatory substance that could activate the complement system through classic or alternative pathways, stimulate mast cells, activate platelets and the coagulation process, and promote blood coagulation. At high concentrations, UA would easily deposit on the small artery layers, thus damaging the intima and increasing the risk for vascular diseases. A high UA concentration might also promote thrombosis through purine metabolism (Nakanishi et al., 2002; Fernández-Real et al., 2003). UA could increase platelet viscosity, urate could destroy the platelets, and UA also plays a role in the formation of free radicals and oxidative stress (Bickel et al., 2002). Diercks et al. (2002) considered that systemic endothelial dysfunction was the common underlying factor of microalbuminuria and macroangiopathy. Currently, scholars believe that microalbuminuria is caused by systemic vascular leakage rather than a specific renal damage (Thomson et al., 2008), and that the accumulation of blood proteins and other macromolecules on blood vessel 
walls would lead to inflammation, lipid deposition, and vascular function disorders, which would eventually lead to atherosclerosis (Kim et al., 2012). Tong et al. (2007) found that patients with T2DM, in association with microalbuminuria, exhibit a significantly higher incidence of macroangiopathy. Therefore, routine clinical monitoring of the FIB, CRP, UA, and UAE levels in diabetic patients and active measures to maintain those indicators within their normal levels would help prevent the occurrence of diabetic LEV.

In brief, HR-CDU has a clinical application in the early detection of diabetic LEV. The patient's age and disease duration are noncontrollable factors of diabetic LEV; however, active promotion of smoking cessation and control of blood pressure, blood sugar, FIB, CRP, $\mathrm{UA}$, and UAE levels could aid in early prevention, thus reducing the mortality and morbidity of LEV.

\section{REFERENCES}

Bao B, Wang Z, Li Y, Weimar W, et al. (2011). The complexities of obesity and diabetes with the development and progression of pancreatic cancer. Biochim. Biophys. Acta 1815: 135-146.

Bickel C, Rupprecht HJ, Blankenberg S, Rippin G, et al. (2002). Serum uric acid as an independent predictor of mortality in patients with angiographically proven coronary artery disease. Am. J. Cardiol. 89: 12-17.

Diercks GF, Van Boven AJ, Hillege JL, de Jong PE, et al. (2002). The importance of microalbuminuria as a cardiovascular risk indicator: a review. Can. J. Cardiol. 18: 525-528.

Engela AU, Baan CC, Dor FJ, Weimar W, et al. (2012). On the interactions between mesenchymal stem cells and regulatory $\mathrm{T}$ cells for immunomodulation in transplantation. Front. Immunol. 3: 126.

Fernández-Real JM, Broch M, Richart C, Vendrell J, et al. (2003). CD14 monocyte receptor, involved in the inflammatory cascade, and insulin sensitivity. J. Clin. Endocrinol. Metab. 84: 1780-1784.

Guo M, Sahni SK, Sahni A and Francis C (2004). Fibrinogen regulates the expression of inflammatory chemokines though NF-кB activity in endothelial cells. Thromb. Haemost. 92: 858-866.

Kanellis J and Kang DH (2005). Uric acid as a mediator of endothelial dysfunction, inflammation, and vascular disease. Semin. Nephrol. 25: 39-42.

Kim HM, Kim KJ, Lee HJ, Yu HT, et al. (2012). Epicardial adipose tissue thickness is an indicator for coronary artery stenosis in asymptomatic type 2 diabetic patients: its assessment by cardiac magnetic resonance. Cardiovasc. Diabetol. 11: 83 .

Makita S, Nakamura M and Hiramori K (2005). The association of C-reactive protein levels with carotid intima-media complex thickness and plaque formation in the general population. Stroke 36: 2138-2142.

Nakanishi N, Sato M, Shirar K, Nakajima K, et al. (2002). Associations between white blood cell count and features of the metabolic syndrome in Japanese male office workers. Ind. Health 40: 273-277.

Pataky Z and Vischer U (2007). Diabetic foot disease in the elderly. Diabetes Metab. 33: S56-65.

Pollanen PJ, Karhunen PJ, Mikkelsson J, Laippala P, et al. (2001). Coronary artery complicated lesion area is related to functional polymorphism of matrix metalloproteinase 9 gene: an autopsy study. Arterioscler. Thromb. Vasc. Biol. 21: $1446-1450$.

Ridker PM, Cushman M, Stampfer MJ, Tracy RP, et al. (1997). Inflammation, aspirin, and the risk of cardiovascular disease in apparently healthy man. N. Engl. J. Med. 336: 973-979.

Seeger FH, Blessing E, Gu L, Bornhold R, et al. (2002). Fibrinogen induces chemotactic activity in endothelial cells. Acta Physiol. Scand. 176: 109-115.

Thomson SE, McLennan SV, Kirwan PD, Heffernan SJ, et al. (2008). Renal connective tissue growth factor correlates with glomerular basement membrane thickness and prospective albuminuria in a non-human primate model of diabetes: possible predictive marker for incipient nephropathy. J. Diabetes Complications 22: 284-294.

Tong PC, Kong AP, So WY, Yang X, et al. (2007). The usefulness of the International Diabetes Federation and the National Cholesterol Education Program's Adult Treatment Panel III definitions of the metabolic syndrome in predicting coronary heart disease in subjects with type 2 diabetes. Diabetes Care 30: 1206-1211.

United Kingdom Prospective Diabetes Study (UKPDS) Group (1998). Tight blood pressure control and risk of macrovascular and microvascular complication in type 2 diabetes. BMJ 317: 703-713.

Valdivielso P, Hidalgo A, Rioja J, Aguilar I, et al. (2007). Smoking and postprandial triglycerides are associated with vascular disease in patients with type 2 diabetes. Atherosclerosis 194: 391-396.

Genetics and Molecular Research 14 (2): 3939-3947 (2015) 
Willigendael EM, Teijink JA, Bartelink ML, Kuiken BW, et al. (2004). Influence of smoking on incidence and prevalence of peripheral arterial disease. J. Vasc. Surg. 40: 1158-1165.

Zhao Z, Nie H, He H, Yan Z, et al. (2007). High-sensitivity C-reactive protein predicts target organ damage in Chinese patients with metabolic syndrome. Metabolism 56: 1612-1619.

Zoccali C, Maio R, Mallamaci F, Sesti G, et al. (2006). Uric acid and endothelial dysfunction in essential hypertension. $J$. Am. Soc. Nephrol. 17: 1466-1471. 